\title{
Accelerating evidence gathering and approval of precision medicine therapies: the FDA takes aim at rare mutations
}

\author{
Amalia M. Issa, PhD, MPH ${ }^{1,2,3}$, Adrian Thorogood, JD ${ }^{3}$, Yann Joly, JD, $\mathrm{PhD}^{3}$ and \\ Bartha M. Knoppers, JD, PhD ${ }^{3}$
}

Genetics in Medicine (2019) 21:542-544; https://doi.org/10.1038/s41436-018-0099-0

Patient heterogeneity in drug response has led to alternative models to the randomized clinical trial (RCT) for drug development, and enabled the evolution of newer regulatory frameworks in recent years. In 2012, the US Food and Drug Administration (FDA) published a popularly used Guidance for Industry on Enrichment Strategies for Clinical Trials to Support Approval Of Human Drugs And Biological Products. ${ }^{1}$ Enrichment strategies refer to the a priori identification and selection of subpopulations of patients, often on the basis of molecular profiling, to increase the likelihood of efficacy of a particular treatment or intervention. ${ }^{1}$ More recently, the FDA released a Draft Guidance for Industry: Developing Targeted Therapies in Low-Frequency Molecular Subsets of a Disease, which provides flexibility to evidence standards to show that a drug works for low-frequency mutations (i.e., "molecular alterations"; strictly for simplicity, we use the short-hand "mutations" in this paper although the Guidance applies to a broad set of molecular alterations). With this new guidance, the FDA proposes to provide sponsors with a strategy for identifying and determining clinical trial eligibility of different patients who have variable molecular modifications. Notably, this new guidance provides broad recommendations for going beyond traditional enrichment strategies, to allow for the grouping of patients having different molecular subtypes of low frequency. The guidance forms part of the FDA's efforts to advance the development and availability of safe and effective targeted treatments for rare diseases, as well as common diseases due to rare mutations. ${ }^{3}$

By focusing on molecular subtypes of low frequency and allowing for the grouping for trial eligibility, the FDA is signaling a change in evidentiary standards. This change has implications not only for pharmaceutical industry sponsors, but also for multiple actors in the drug development process. In this paper, we examine the impact of these changing evidentiary standards on the conduct of clinical trials, drug approvals and prescriber duties, and patients, as well as the need for data sharing.

\section{POSSIBLE IMPACT ON CLINICAL TRIALS}

The FDA Guidance states, "clinical trial assays should be designed to detect all possible molecular alterations that comprise the group that is expected to respond." ${ }^{3}$ Typically, in an enrichment design, a strong biomarker that can identify a subgroup of patients for whom the treatment is expected to be particularly effective allows for the enrollment of a small sample size that is adequately powered to detect an appropriate treatment effect (e.g., in phase III studies 0.025 for a one-sided statistical significance). An accepted concept in precision medicine (including by FDA authors) is agreement that within a clinical study, a predictive biomarker should be able to distinguish between a subgroup of patients who will benefit (biomarker positive) from those who will not benefit (biomarker negative) from treatment., 5

The new FDA draft guidance explicitly cautions sponsors to use analytically valid assays; however, no discussion is provided about clinical validity concerns that might arise in the course of using biomarkers to identify molecular alterations of low frequency.

The FDA acknowledges, "low numbers...would in most settings preclude meaningful empirical inferences about treatment benefits or risks...." ${ }^{3}$ Nevertheless, the new Guidance presumes that "extrapolation across multiple subsets may be possible despite the low frequency or absence of patients in some subsets." ${ }^{3}$ The new evidence standards thus appear to favor internal validity over external validity, but do not address explicitly how sponsors are to interpret this departure from the accepted standards of evidence and rigorous RCT design and conduct. This presents challenges given that many stakeholders rely on RCTs for evidence of clinical validity and utility.

${ }^{1}$ Personalized Medicine \& Targeted Therapeutics, University of the Sciences in Philadelphia, Philadelphia, Pennsylvania, USA; ${ }^{2}$ Pharmaceutical Sciences; Health Policy \& Public
Health, University of the Sciences in Philadelphia, Philadelphia, Pennsylvania, USA; ${ }^{3}$ Centre of Genomics and Policy, McGill University, Montreal, Quebec, Canada. Health, University of the Sciences in Philadelphia, Philad
Correspondence: Amalia M. Issa (amalia.issa2@mcgill.ca)

Submitted 9 February 2018; accepted: 7 June 2018

Published online: 10 July 2018 


\section{THE NEED FOR DATA SHARING}

The creation and integration of more data to support lowfrequency molecular drug indications will require new collaborative approaches to data generation by researchers, clinical laboratories, and genetic databases. The need for data sharing is evident both upstream (to support clinical trial inclusion criteria) and downstream (enhanced pharmacosurveilllance) of approval. Upstream, it is unlikely that sponsors will be able to generate data on rare mutations on their own. For example, the approach pioneered for the cystic fibrosis transmembrane conductance regulator (CFTR) gene depended, in part, on evidence from a genetic database, which was progressively populated by researchers and clinical laboratories. ${ }^{6}$ Higher standards for genetic databases will need to be developed and perhaps even regulated, to ensure the quality of data. More reliance on external databases by sponsors can raise concerns about database transparent aggregation, curation and interpretation of variants, data quality, and possible conflicts of interest. Indeed, previous FDA draft guidance initiated discussions on establishing standards, or even certification, for variant databases.

With drugs approved with lower evidentiary standards, the FDA Guidance also calls for enhanced postapproval patient surveillance. While clinical data sharing is an emerging norm for clinicians and laboratories, ${ }^{7}$ both the FDA and sponsors will need to take a greater leadership role in promoting and funding data sharing. Including rare mutations on drug labels will support more rigorous patient monitoring and data sharing than the current use of off-label prescribing.

\section{DRUG LABELING AND PRESCRIBERS' DUTIES TO PATIENTS}

To offset the lower evidentiary standard, the FDA draft guidance calls for detailed labeling information to accompany drugs, including information about the level of evidence supporting rare molecular indications. Stakeholders, such as prescribers and payers, traditionally work on the assumption that indications, including molecular indications, are based on clinical trial data. The FDA guidance calls for labels to include a transparent description of the (limited) evidentiary basis for rare molecular indications. While commendable, this shift will demand greater scientific literacy and accountability on the part of health professionals prescribing the drug.

Because so few medicines have been approved specifically to treat rare diseases, ${ }^{8}$ there is a significant amount of off-label use in rare diseases. This status quo puts significant responsibility on clinicians. Off-label use might be the standard of care for many patients who are in dire need of new drugs to manage their rare condition. However, off-label prescribing may expose physicians to legal claims of medical malpractice if informed consent is insufficient or if sound medical judgment, based on the available evidence, ${ }^{9}$ was not properly exercised. Furthermore, it is possible that the new Guidance would require that patients be tested for the relevant biomarkers, a practice that is not routinely carried out with off-label prescribing.
The Guidance could help to ensure that some (as opposed to no) evidence is generated in clinical trials for rare mutations. In addition, this approach could help inform prescriber and patient shared decision-making in selecting a therapy. One caveat, however, is that prescribers will need to explain complex labels, that drugs are "indicated," but that this indication is supported only by limited evidence.

\section{IMPACT ON PATIENTS}

The new Guidance may change clinical practice and impact patient care in addition to the effects of changing drug labels and prescriber duties. Particularly salient is the question of how likely it is that a new treatment, if approved under these new evidentiary standards, would be used in clinical practice, given that clinicians typically use RCTs for evidence of clinical validity and utility. Accepting groupings of patients with particular molecular variations into a given clinical study may be a windfall for industry sponsors looking to identify the right patient for the drug. However, within the real world context of clinical practice, this may be less useful in identifying the right drug for the patient, which is ultimately the desired health outcome. Nevertheless, the new Guidance provides opportunities to further clinical research on lowfrequency mutations for both rare and more common diseases, and this may ultimately be beneficial for patients, who have hitherto been underserved with respect to targeted treatments.

\section{CONCLUSION}

The FDA is leading international efforts to encourage the adoption of precision medicine, and to improve accessibility of drugs for individuals with rare disease. What does this change ultimately mean for patients with rare diseases? The Draft Guidance may improve incentives for sponsors to run small trials, ultimately improving the evidence base and therapeutic options for these patients. It might encourage sponsors and regulators to determine molecular indications according to standard albeit lowered evidentiary standards, rather than putting the onus on clinicians to rely on "offlabel" use. Indeed, what was once an off-label use might, with the new Guidance, become an on-label use, with any adverse outcomes reported through postmarket surveillance mechanisms. Success will depend on innovation by, and collaboration between, multiple stakeholders, including prescribers, regulators, payers, and patients.

\section{DISCLOSURE}

The authors declare no conflict of interest.

\section{REFERENCES}

1. US Food and Drug Administration. Guidance for industry. Enrichment strategies for clinical trials to support approval of human drugs and biological products. 2012. http://www.fda.gov/downloads/Drugs/ GuidanceComplianceRegulatoryInformation/Guidances/UCM332181.pdf. Accessed January 8, 2018.

2. US Food and Drug Administration. Guidance for industry developing targeted therapies in low-frequency molecular subsets of a disease 
guidance for industry. December 2017 https://www.fda.gov/downloads/ Drugs/GuidanceComplianceRegulatoryInformation/Guidances/ UCM588884.pdf. Accessed December 20, 2017.

3. US Food and Drug Administration. Statement from FDA Commissioner Scott Gottlieb, M.D., on new FDA efforts to support more efficient development of targeted therapies. December 15, 2017. https://www. fda.gov/NewsEvents/Newsroom/PressAnnouncements/ucm589248.htm. Accessed December 20, 2017.

4. Amur S, LaVange L, Zineh I, Buckman-Garner S, Woodcock J. Biomarker qualification: toward a multiple stakeholder framework for biomarker development, regulatory acceptance, and utilization. Clin Pharmacol Ther. 2015;98:34-46.

5. Saad ED, Paoletti X, Burzykowski T, Buyse M, Precision medicine needs randomized clinical trials. Nat Rev Clin Oncol. 2017;14:317-23.

6. Tsui L-C, Dorfman R. The cystic fibrosis gene: a molecular genetic perspective. Cold Spring Harb Perspect Med. 2013;3:a009472.

7. ACMG Board of Directors. Laboratory and clinical genomic data sharing is crucial to improving genetic health care: a position statement of the American College of Medical Genetics and Genomics. Genet Med. 2017:19:721-2.

8. Austin $C P$, Cutillo $C M$, Lau LPL, et al. Future of rare diseases research 2017-2027: an IRDiRC perspective. Clin Transl Sci. 2017;11:21-7.
9. Eguale B, Buckeridge DL, Verma A, et al. Association of off-label drug use and adverse drug events in an adult population. JAMA Intern Med. 2016;176:55-63.

(i) (-) Open Access This article is licensed under a Creative Commons (C) Attribution-NonCommercial-NoDerivatives 4.0 International License, which permits any non-commercial use, sharing, distribution and reproduction in any medium or format, as long as you give appropriate credit to the original author(s) and the source, and provide a link to the Creative Commons license. You do not have permission under this license to share adapted material derived from this article or parts of it. The images or other third party material in this article are included in the article's Creative Commons license, unless indicated otherwise in a credit line to the material. If material is not included in the article's Creative Commons license and your intended use is not permitted by statutory regulation or exceeds the permitted use, you will need to obtain permission directly from the copyright holder. To view a copy of this license, visit http://creativecommons.org/licenses/by-nc-nd/4.0/.

(C) The Author(s) 2018 